\title{
Longitudinal Changes in Health-Related Quality of Life for Chronic Diseases: An Example in Hemophilia A
}

\author{
Jiat-Ling Poon, PhD, Jason N. Doctor, PhD, and Michael B. Nichol, PhD \\ University of Southern California, School of Pharmacy, Los Angeles, CA, USA.
}

\begin{abstract}
BACKGROUND: Patients with well-managed rare chronic diseases such as hemophilia maintain a stable health state and health-related quality of life (HrQoL) that may be affected by acute events. Longitudinal HrQoL assessments analyzed using multivariate multilevel (MVML) modelling can determine the impact of such events on individuals (within-person effect) and identify factors influencing within-population differences (between-person effect).
\end{abstract}

OBJECTIVES: To demonstrate the application of MVML modelling in a longitudinal study of $\mathrm{HrQoL}$ in hemophilia A.

METHODS/DESIGN/PARTICIPANTS: Using data on 136 adults and 125 children from a two-year observational cohort study of burden of illness in US hemophilia A patients, MVML modelling determined the effect of time-invariant (sociodemographic and clinical characteristics) and time-varying factors (bleeding frequency, emergency room visits, and missed work/school days) on within-person and between-person HrQoL changes. HrQoL was assessed using the SF-12 health survey (adults) and PedsQL inventory (children) at baseline, then every 6 months.

RESULTS: In children, within-person $(p<0.0001)$ and between-person $(p<0.0001)$ psychosocial functioning was reduced by each additional bleed and missed day (within-person: $p=0.0089$; between-person: $p=0.0060$ ). Within-person physical functioning was reduced by each additional bleed $(p<0.0001)$, emergency room (ER) visit $(p=0.0284)$, and missed day $(p=0.0473)$. Between-persons, additional missed days $(p<0.0001)$ significantly decreased physical functioning. In adults, each additional missed day reduced SF-12 Health Survey mental $(p=0.0025)$ and physical $(p=0.0093)$ component summary scores. Each additional bleed also decreased physical component summary (PCS) significantly ( $p=0.0093)$.

CONCLUSIONS: This study demonstrated the applicability of MVML modelling in identifying time-invariant and time-varying factors influencing $\mathrm{HrQoL}$ in a rare chronic disease population. Small but significant within-person and between-person changes in HrQoL with each additional acute event experienced were identified, which if frequent, could have a large cumulative impact. The results suggest that MVML modelling may be applied to future studies of longitudinal change in $\mathrm{HrQoL}$ in other rare chronic disease populations.

Published online July 17, 2014
KEY WORDS: health-related quality of life; hemophilia A; longitudinal; chronic disease; rare disease.

J Gen Intern Med 29(Suppl 3):S760-6

DOI: $10.1007 / \mathrm{s} 11606-014-2893-y$

(c) Society of General Internal Medicine 2014

\section{INTRODUCTION}

In stable chronic disease, well-managed patients typically maintain a relatively constant health state over a period of time, as measured by disease-specific clinical indicators. Patient-reported outcomes (PROs) such as health-related quality of life (HrQoL) are also expected to remain relatively stable. Thus, observing the same patient at two discrete time points might lead to conclusions that there were no significant changes in clinical outcomes or PROs over time.

Although an individual patient's clinical condition is not expected to deviate significantly over time from a chronic baseline, fluctuations are expected in response to acute clinical exacerbations or external changes. Those in turn lead to within-person deviations in HrQoL from the chronic baseline. While it is important to be able to identify withinperson fluctuations in $\mathrm{HrQoL}$, they do not necessarily generalize to any between-person differences among individuals with the same disease.

To identify such within-person and between-person effects of factors influencing HrQoL, repeated measurements of the same patients must be made across a period of time. Even if a significant pattern of growth or decline over time is not demonstrated, longitudinal data allow for the disaggregation of within-person and between-person effects. Analytical methods like multivariable multilevel modelling (MVML) can be employed to disaggregate these effects, helping researchers and healthcare providers understand the factors mediating $\mathrm{HrQoL}$, allowing the latter to better manage patient care both at the levels of the individual patient and the disease population.

To illustrate the partitioning of between-person and within-person effects of clinical measures or symptom manifestations on HrQoL over time, we consider a sample of persons with hemophilia A, a rare chronic sex-linked 
disease primarily affecting males, characterized by a deficiency in clotting factor VIII that results in the inability of the blood to clot normally. Although patients are typically asymptomatic and are able to lead active and productive lives, ${ }^{1}$ they may experience acute bleeding episodes either spontaneously or in the rare event of trauma. Repeated bleeding into the joints may eventually lead to debilitating chronic hemophilic arthropathy. ${ }^{2}$ To prevent this, persons with hemophilia A are treated with factor replacement therapy, either as regular prophylactic infusions of clotting factor concentrates or on-demand during acute bleeding episodes. We expect that hemophilia A patients will have stable HrQoL, except for when there is transient worsening during acute symptom exacerbations.

Several studies have previously captured longitudinal $\mathrm{HrQoL}$ data in persons with bleeding disorders, including hemophilia A. However, none has attempted to describe the effects of changes in the frequency of symptoms or clinical status on HrQoL. ${ }^{3-5}$ Over 18 months, Gringeri et al. ${ }^{3}$ found that $\mathrm{HrQoL}$ remained stable in a group of 41 hemophilia $\mathrm{A}$ and $\mathrm{B}$ patients with inhibitors when measured at 6-month intervals; they therefore reported only baseline $\mathrm{HrQoL}$ results. Similarly, over a 5-year period with annual follow-up, Lindvall et al. ${ }^{4}$ found no statistically significant changes in HrQoL among a group of ten patients with severe hemophilia. Solovieva ${ }^{5}$ observed a statistically significant improvement in physical functioning in mild and moderate hemophilia patients and a statistically significant reduction in vitality in severe hemophilia patients on the SF-36 scale over 3 years. However, that study gathered data at only two time points (year zero and 3 years later), and was not designed to capture possible fluctuations in HrQoL between those two points.

In rare diseases such as hemophilia, where study sample sizes are typically small (as demonstrated above), the use of longitudinal data increases the number of observations available for analysis, allowing for trends or influencing factors on the outcome of interest to be identified. Using data from the Hemophilia Utilization Group Study Part Va (HUGS Va), the objective of the current analysis was to demonstrate the application of multivariate multilevel modelling (MVML) in a population with a rare disease such as hemophilia, among whom HrQoL is hypothesized to vary over time between persons, as well as to fluctuate within persons.

\section{METHODS}

\section{Data}

The Hemophilia Utilization Group Study Part Va (HUGS Va) was a two-year multicenter observational cohort study conducted among hemophilia A patients from six hemophilia treatment centers (HTCs) in geographically diverse regions of the US. Details of the study methods and inclusion criteria have previously been reported. ${ }^{6},{ }^{7}$ Informed consent and data were collected at initial interview from participant/parent selfor proxy-report and patient chart reviews completed by healthcare providers. Information regarding clinical aspects of the disease, such as treatment regimen, arthropathy and comorbidities, as well as $\mathrm{HrQoL}$ and the economic consequences of having hemophilia A, were collected. Periodic participant follow-up surveys collected data on time lost from work, school, or usual activities, disability days, healthcare utilization and clinical outcomes, including bleeding episodes, joint pain, and motion limitation. Follow-up interviews were administered monthly in the first year and semi-annually in the second year. HrQoL was measured at baseline and every 6 months during the 2-year follow-up. Baseline HrQoL and its association with joint pain and motion limitation in the HUGS Va population has previously been reported. ${ }^{7}$ The current analysis includes data from 261 participants (136 adults and 125 children) who completed their initial interview and at least one follow-up, out of a total of 329 participants (164 adults and 165 children) with factor VIII deficiency who were recruited into the HUGS Va study between July 2005 and July 2007.

The University of Southern California (USC) served as the data and coordinating center. The study protocol was approved by the Institutional Review Board of USC (IRB number: HS-046012) and that of each participating HTC.

\section{HrQoL Instruments}

Adult general health was assessed using the SF-12 Health Survey Version 1, which has been used in previous hemophilia studies. ${ }^{6-10}$ The physical component summary (PCS) and mental component summary (MCS) scores were used in this analysis. ${ }^{11} \mathrm{HrQoL}$ of participants aged 2-17 years was assessed by parent-proxy using the PedsQL ${ }^{\mathrm{TM}} 4.0$ generic core scales, ${ }^{12}$ a HrQoL instrument developed in the US for children and adolescents. The PedsQL has been shown to be valid and reliable for both self and parent-proxy administration, and is also sensitive to differences between disease types and between severity groups within the same disease. ${ }^{12-14} \mathrm{In}$ this analysis, the physical and psychosocial health summary scores of the PedsQL were used.

Generic HrQoL instruments were used instead of a hemophilia-specific tool to allow for comparison with other disease populations. Additionally, generic instruments were used, because hemophilia-specific HrQoL instruments validated for use in the US population were not available at the time of study initiation.

\section{Analysis}

HrQoL was measured at $0,6,12,18$, and 24 months. Baseline (0 month) and measurements obtained within a 6month window (3 months pre and post) of each follow-up 
time point were included for analysis. The outcomes of interest for adults were the SF-12 PCS and MCS. For children, the outcomes of interest were the two summary scores of physical health and psychosocial health from the PedsQL.

To disaggregate within-person and between-person effects of patient characteristics and outcomes on HrQoL, both time-varying and time-invariant covariates need to be considered. Time-varying covariates were the frequency of bleeding episodes, emergency room visits, and missed days of work or school due to hemophilia. For each event, the total number of occurrences between each follow-up period (as determined by the time between each HrQoL measurement) were summed and attributed to that period. Timeinvariant covariates were hemophilia severity, treatment type, patient or parent employment status, and health insurance coverage.

Multivariate multilevel (MVML) modelling was performed using SAS 9.3 (SAS Institute Inc., Cary, NC, USA) to characterize longitudinal within-person and between-person variations in HrQoL. Data from longitudinal studies such as HUGS Va have a hierarchical structure, under which measurements at each follow-up time point are nested within individual subjects. Because repeated measurements from the same subjects are involved, the correlation of residuals in the model must be controlled. Multilevel models represent the data in hierarchical levels, controlling residual correlations and eliminating the need to aggregate data or analyze each level separately. At the same time, multilevel models estimate each individual subject's growth trajectory (within-person effects), while also determining how individual growth parameters vary as a function of differences between subjects in background characteristics (between-person effects). ${ }^{15},{ }^{16}$

The analysis is conceptualized in two stages. In stage 1, the within-person model is considered, in which the HrQoL of a participant is a function of his HrQoL change over time plus random error. ${ }^{15-17}$ The time-varying covariates for each participant is centered (difference between frequency of event at each time point and the participant's cross-time average) on his cross-time average (mean frequency of event for each participant in each 6-month period), representing the rate of change of HrQoL with every unit increase in the covariate above the individual-mean at each time point. Including the time-varying covariates of interest in this stage of the analysis estimates the within-person effects of these covariates. Because they are constant within each participant, time-invariant covariates do not need to be included in this stage of the analysis. In stage 2, we are interested in the between-person variations of HrQoL as a function of clinical or sociodemographic differences. These would include the time-invariant covariates and the grandmean (mean frequency for the entire study population across two years)-centered cross-time averages of each participant's time-varying covariates. The interaction between hemophilia severity and treatment type was also included in the models, and the effects of interactions between the main effects of interest (described above) on HrQoL were also explored. The reduced equation model combining stages 1 and 2 was fitted using the SAS PROC MIXED command, with repeated measurements for each subject, allowing the means to vary randomly across individuals and assuming random effects of time.

\section{Addressing Missing Data}

To account for missing data due to loss of follow-up in both the outcomes of interest (HrQoL scores) and timevarying covariates of interest, multiple imputation was employed using IVEware version 0.2 (Survey Methodology Program, Survey Research Center, Institute for Social Research, University of Michigan). ${ }^{18}$ A multivariate imputation procedure was performed sequentially on each covariate of interest, conditioned on the subject's age and race as well as all observed time-varying variables. Imputed continuous variables are assumed to follow a normal distribution, and count variables are assumed to follow a Poisson distribution.

\section{RESULTS}

Of the HUGS Va study population, 261 participants (136 adults, 125 children) had baseline and at least one follow-up HrQoL measurement. At baseline, the mean ages of adults and children in the study were $33.1 \pm 12.0$ years and $9.6 \pm$ 4.4 years, respectively. The majority of the study population was White/Non-Hispanic (75.0 \% adults; $68.0 \%$ children) and had severe hemophilia A (64.7\% adults; $67.2 \%$ children). Across all severities, $42.2 \%$ of patients received prophylactic factor replacement therapy, of which $61.6 \%$ (34 adults and 72 children) had severe hemophilia. Most participants or their parents (for children) had part-time or full-time employment ( $62.5 \%$ of adult participants; $73.6 \%$ of parents of child participants) and had some form of health insurance (91.9\% adults; $100 \%$ children) (Table 1).

Over two years, there were wide variations between each 6-month follow-up period in mean number of bleeding episodes, emergency room (ER) visits, and missed days of work or school due to hemophilia A (Table 2). Mean MCS and PCS for the entire adult study population at baseline were $50.8 \pm 10.2$ and $43.0 \pm 10.8$, respectively, remaining fairly constant throughout follow-up (Fig. 1). Mean psychosocial and physical functioning scores for the pediatric study population were $83.0 \pm 15.7$ and $88.0 \pm 16.4$, respectively, with greater variation in physical functioning scores than psychosocial functioning scores over time (Fig. 2). 
Table 1. Baseline Demographics

\begin{tabular}{llll}
\hline \hline Variables, N (\%) & $\begin{array}{l}\text { All } \\
\text { Patients } \\
(\mathbf{N}=\mathbf{2 6 1})\end{array}$ & Adults & Children \\
& $\mathbf{N}=\mathbf{1 3 6})$ & $\mathbf{( N = 1 2 5 )}$ \\
\hline Mean Age at Baseline (SD) & $21.8(15.0)$ & $33.1(12.0)$ & $9.6(4.4)$ \\
Race & $187(71.7)$ & $102(75.0)$ & $85(68.0)$ \\
$\quad$ White & $74(28.3)$ & $34(25.0)$ & $40(32.0)$ \\
$\quad$ Other & & & \\
Hemophilia Severity & $89(34.1)$ & $48(35.3)$ & $41(32.8)$ \\
$\quad$ Mild/Moderate & $172(65.9)$ & $88(64.7)$ & $84(67.2)$ \\
$\quad$ Severe & $110(42.2)$ & $36(26.5)$ & $74(59.2)$ \\
Treatment Type & $151(57.8)$ & $100(73.5)$ & $51(40.8)$ \\
$\quad$ Prophylaxis & $174(66.7)$ & $82(60.3)$ & $92(73.6)$ \\
$\quad$ On-Demand & $76(29.1)$ & $43(31.6)$ & $33(26.4)$ \\
Health Insurance Type & $11(4.2)$ & $11(8.1)$ & $0(0.0)$ \\
$\quad$ Private Insurance & $116(44.4)$ & $60(44.1)$ & $56(44.8)$ \\
$\quad$ Public Insurance & $61(23.4)$ & $25(18.4)$ & $36(28.8)$ \\
$\quad$ No Insurance & $84(32.2)$ & $51(37.5)$ & $33(26.4)$ \\
Employment Status* & & & \\
$\quad$ Full-time & Part-time & &
\end{tabular}

*Employment status of adults and parents of children $\leq 17$ years of age

Table 3 shows the results of the final MVML models for the adult study population, with separate models constructed for the MCS and PCS. Employment status was found to have a significant influence on MCS ( $p=0.0160$ for fulltime employed vs. unemployed), as did between-person differences in missed work/school days $(p=0.0026)$ and within-person changes in bleeding frequency $(p=0.0243)$ and ER visits $(p=0.0350)$. Only significant interactions between the main effects of interest were included in the final models. MCS was significantly moderated by the interactions of employment status with within-person changes in bleeding episodes ( $p=0.0069$ for part-time vs. unemployed), employment with within-person changes in missed days ( $p=0.0309$ for full-time vs unemployed), insurance status with within-person changes in ER visits ( $p=0.0160$ for no insurance vs. public insurance), and within-person changes in missed days with hemophilia severity $(p=0.0161)$. PCS was significantly influenced by insurance status $(p=0.0047$ and $p=0.0007$ for full-time and

Table 2. Study Population Grand Means Over Time

\begin{tabular}{clll}
\hline $\begin{array}{c}\text { Follow-up Period } \\
\text { (Mean } \pm \text { SD) }\end{array}$ & $\begin{array}{l}\text { Number } \\
\text { of Bleeds }\end{array}$ & ER Visits & $\begin{array}{l}\text { Missed Days } \\
\text { of Work/School }\end{array}$ \\
\hline Adults $(\mathrm{N}=136)$ & & & \\
6 months & $10.4 \pm 14.8$ & $0.9 \pm 1.4$ & $12.0 \pm 33.9$ \\
12 months & $8.0 \pm 10.5$ & $0.5 \pm 1.2$ & $11.8 \pm 38.0$ \\
18 months & $10.1 \pm 15.7$ & $0.4 \pm 0.8$ & $6.9 \pm 21.3$ \\
24 months & $6.5 \pm 11.6$ & $0.3 \pm 0.8$ & $5.2 \pm 23.3$ \\
Children $(\mathrm{N}=125)$ & & & \\
6 months & $4.8 \pm 10.7$ & $0.6 \pm 1.6$ & $2.5 \pm 4.7$ \\
12 months & $3.8 \pm 6.7$ & $0.3 \pm 1.1$ & $4.8 \pm 17.9$ \\
18 months & $4.7 \pm 12.2$ & $0.5 \pm 1.3$ & $3.6 \pm 13.2$ \\
24 months & $7.1 \pm 13.5$ & $0.3 \pm 0.8$ & $4.5 \pm 8.6$ \\
\hline
\end{tabular}

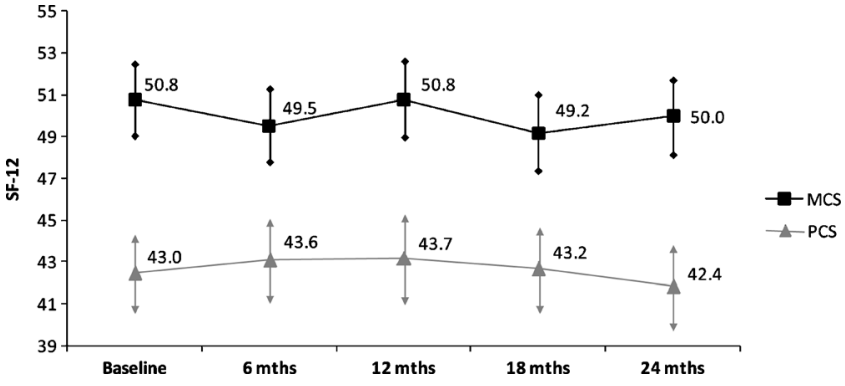

Figure 1. Adults-Study population mean and $95 \%$ confidence interval* for follow-up HrQoL scores $(N=136)$. *Arrows denote $95 \%$ confidence interval.

part-time employed vs. unemployed, respectively), hemophilia treatment type $(p=0.0424)$, both between-person and within-person variations in missed work/school days (between-person: $p<0.0001$; within-person: $p=0.0064$ ), and between-person differences in bleeding frequency $(p=0.0093)$. The interaction of between-person differences in missed days with within-person changes in bleeding episodes $(p=0.0223)$, as well as that of between-person differences in bleeding episodes with within-person changes in ER visits $(p=0.0046)$ significantly influenced PCS. The results suggest that frequent bleeds and missed days may affect the ability of patients to maintain active and productive lifestyles, ultimately affecting HrQoL as well.

Table 4 shows the results of the final MVML model for the pediatric study population. Hemophilia severity $(p=0.0139)$, insurance status $(p=0.0047)$, and parental employment status ( $p=0.0496$ for full-time vs. unemployed) had a significant influence on psychosocial functioning. Between-person $(p<0.0001)$ and within-person $(p=0.0013)$ variations in bleeding frequencies and both between-person $(p=0.0043)$ and within-person $(p=0.0023)$ variations in days missed from school/work also had significant impacts on psychosocial functioning. Several interaction terms were also found to moderate psychosocial functioning. These included

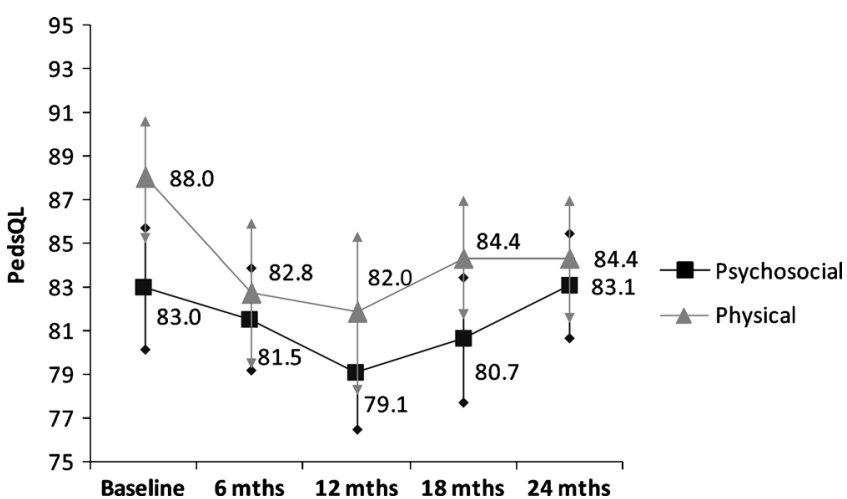

Figure 2. Children-Study population mean and $95 \%$ confidence interval* for follow-up HrQoL scores $(\mathrm{N}=125)$. *Arrows denote $95 \%$ confidence interval. 
Table 3. Adults-Results from multivariate multilevel model $(\mathrm{N}=136)$

\begin{tabular}{|c|c|c|c|c|c|c|}
\hline & \multicolumn{3}{|c|}{$\begin{array}{l}\text { Mental Component Score } \\
\text { (MCS) }\end{array}$} & \multicolumn{3}{|c|}{$\begin{array}{l}\text { Physical Component Score } \\
\text { (PCS) }\end{array}$} \\
\hline & $\beta$ & SE & p value & $\beta$ & SE & p value \\
\hline Intercept & 47.4 & 2.0 & & 35.7 & 2.0 & \\
\hline Time & -0.1 & 0.2 & 0.6355 & -0.1 & 0.2 & 0.5383 \\
\hline Hemophilia Severity: Mild/Moderate & 9.3 & 5.8 & 0.1093 & -6.8 & 5.8 & 0.2424 \\
\hline Treatment Type: On-demand Therapy & 1.2 & 1.9 & 0.5378 & 2.1 & 1.9 & 0.2613 \\
\hline \multicolumn{7}{|l|}{ Insurance $($ Ref $=$ Public) } \\
\hline Private Insurance & 1.5 & 1.8 & 0.4004 & 3.8 & 1.8 & 0.0322 \\
\hline No Insurance & -0.6 & 2.9 & 0.8240 & -0.2 & 2.9 & 0.9575 \\
\hline \multicolumn{7}{|l|}{ Employment Status (Ref = Unemployed) } \\
\hline Full-time & -0.7 & 1.9 & 0.0160 & 5.3 & 1.9 & 0.0047 \\
\hline Part-time & 5.1 & 2.1 & 0.7217 & 7.2 & 2.1 & 0.0007 \\
\hline \multicolumn{7}{|l|}{ Between-Person Effects } \\
\hline Bleeding Episodes & 0.008 & 0.09 & 0.9250 & -0.2 & 0.09 & 0.0093 \\
\hline ER Visits & -1.4 & 1.4 & 0.3063 & -0.1 & 1.3 & 0.9427 \\
\hline Days missed from work/school & -0.1 & 0.04 & 0.0026 & -0.2 & 0.04 & $<0.0001$ \\
\hline \multicolumn{7}{|l|}{ Within-Person Effects } \\
\hline Bleeding Episodes & -0.00004 & 0.05 & 0.9993 & -0.04 & 0.03 & 0.2170 \\
\hline ER Visits & 0.3 & 0.4 & 0.4817 & 0.3 & 0.3 & 0.3652 \\
\hline Days missed from work/school & -0.003 & 0.02 & 0.8603 & -0.04 & 0.01 & 0.0064 \\
\hline \multicolumn{7}{|l|}{ Interactions of Interest } \\
\hline Severity and Treatment (Severe hemophilia and receiving prophylaxis) & -8.1 & 6.0 & 0.1796 & 7.9 & 6.0 & 0.1888 \\
\hline \multicolumn{7}{|l|}{ Employment and $\mathrm{W} /$ in Bleeds } \\
\hline Full-time & 0.01 & 0.07 & 0.8818 & - & - & - \\
\hline Part-time & -0.3 & 0.1 & 0.0069 & - & - & - \\
\hline \multicolumn{7}{|l|}{ Employment and $\mathrm{W} / \mathrm{in}$ Missed days } \\
\hline Full-time & -0.1 & 0.1 & 0.0309 & - & - & - \\
\hline Part-time & 0.03 & 0.03 & 0.4391 & - & - & - \\
\hline \multicolumn{7}{|l|}{ ER Visits and Insurance } \\
\hline Private Insurance & -0.9 & 0.6 & 0.1718 & - & - & - \\
\hline No Insurance & -3.2 & 1.3 & 0.0160 & - & - & - \\
\hline \multicolumn{7}{|l|}{ Days Missed and Severity: } \\
\hline Mild/Moderate Hemophilia & 0.09 & 0.04 & 0.0161 & - & - & - \\
\hline Between-person missed days and within-person bleeding episodes & - & - & - & -0.0003 & 0.001 & 0.0223 \\
\hline Between-person bleeding episodes and within-person ER visits & - & - & - & 0.07 & 0.03 & 0.0046 \\
\hline
\end{tabular}

Italicised values are significant at the 0.05 level

the interaction of disease severity with treatment type ( $p=$

0.0404), parental employment status with within-person changes in patient missed days ( $p=0.0035$ for full-time vs. unemployed), and between-person differences in bleeding

Table 4. Children-Results from Multivariate Multilevel Model (N=125)

\begin{tabular}{|c|c|c|c|c|c|c|}
\hline & \multicolumn{3}{|c|}{ Psychosocial Functioning } & \multicolumn{3}{|c|}{ Physical Functioning } \\
\hline & $\beta$ & SE & p value & $\beta$ & SE & p value \\
\hline Intercept & 75.4 & 3.1 & & 82.5 & 3.3 & \\
\hline Time & 0.2 & 0.3 & 0.4069 & -0.2 & 0.3 & 0.4675 \\
\hline Hemophilia Severity: Mild/Moderate & -8.8 & 3.5 & 0.0139 & -2.4 & 3.7 & 0.5239 \\
\hline Treatment Type: On-demand Therapy & -10.7 & 7.3 & 0.1421 & -7.0 & 7.6 & 0.3565 \\
\hline \multicolumn{7}{|l|}{ Insurance $($ Ref $=$ Public $)$} \\
\hline Private Insurance & 6.4 & 2.2 & 0.0047 & 4.5 & 2.4 & 0.0588 \\
\hline \multirow{2}{*}{\multicolumn{7}{|c|}{ Parental Employment Status }} \\
\hline & & & & & & \\
\hline $\begin{array}{l}\text { Full-time } \\
\text { Part-time }\end{array}$ & -4.5 & 2.3 & 0.0496 & 1.0 & 2.4 & 0.6868 \\
\hline Part-time & -0.4 & 2.5 & 0.8754 & 4.2 & 2.6 & 0.1117 \\
\hline \multicolumn{7}{|l|}{ Between-Person Effects } \\
\hline Bleeding Episodes & -0.8 & 0.2 & $<0.0001$ & -0.2 & 0.2 & 0.2425 \\
\hline ER Visits & -0.7 & 1.4 & 0.6025 & 0.04 & 1.4 & 0.9804 \\
\hline Days missed from work/school & -0.6 & 0.2 & 0.0043 & -1.4 & 0.2 & $<0.0001$ \\
\hline \multicolumn{7}{|l|}{ Within-Person Effects } \\
\hline Bleeding Episodes & -0.2 & 0.1 & 0.0013 & -0.5 & 0.2 & 0.0035 \\
\hline ER Visits & -0.4 & 0.4 & 0.3611 & -1.4 & 0.5 & 0.0095 \\
\hline Days missed from work/school & -0.7 & 0.2 & 0.0019 & -0.3 & 0.2 & 0.0666 \\
\hline Interactions of Interest & & & & & & \\
\hline Severity and Treatment (Severe hemophilia and receiving prophylaxis) & -16.4 & 8.0 & 0.0404 & -14.2 & 8.4 & 0.0909 \\
\hline \multicolumn{7}{|l|}{ Parental Employment and W/in Missed Days } \\
\hline Full-time & 0.7 & 0.3 & 0.0035 & - & - & - \\
\hline Part-time & -0.3 & 0.3 & 0.3955 & - & - & - \\
\hline Between-person bleeding episodes and within-person missed days & 0.06 & 0.02 & 0.0003 & - & - & - \\
\hline Within-person bleeding episodes and Severity: Mild/Moderate & - & - & - & -0.4 & 0.2 & 0.0125 \\
\hline
\end{tabular}


episodes and within-person changes in patient missed days $(p=0.0003)$. Physical functioning was significantly influenced by between-person differences in missed days from school/work $(p<0.0001)$ and by within-person changes in bleeding frequencies $(p=0.0031)$ and ER visits $(p=0.0095)$. The interaction of within-person changes in bleeding frequencies with hemophilia severity also significantly influenced physical functioning $(p=0.0125)$. In children, psychosocial functioning was affected to a greater extent than physical functioning, suggesting that acute events restrict the activities of children, reducing their social interactions with peers and affecting them psychosocially.

\section{DISCUSSION}

This study demonstrated the application of a multivariable multilevel (MVML) model to a population with a rare disease, namely hemophilia. Employing MVML modelling allowed for the disaggregation of both between-person and within-person changes in HrQoL. Small but significant changes with each additional acute event (bleeds/ER visits/ missed days) were identified, which individually decreased mental or physical HrQoL by only a small amount. However, frequent events within a particular time period could have a large cumulative impact on patients' HrQoL, yielding clinically important differences in $\mathrm{HrQoL}$, either within-person across time periods or between-persons. Between persons, the MVML model also allowed for the identification of sociodemographic differences between individuals that could affect HrQoL, as well as interactions between sociodemographic characteristics and the frequency of acute events that could moderate HrQoL. Using MVML modelling, our analysis showed the additive effects of sociodemographic differences between patients, frequency of acute events, and their interactions on the HrQoL of individual patients, both relative to others with hemophilia as well as to themselves over time.

This analysis was restricted by the small study sample size of 136 adults and 125 children. However, these are typical sample sizes in studies of rare diseases, and to our knowledge HUGS Va is one of the larger studies conducted in a population with hemophilia A. Nevertheless, small sample sizes present difficulties when selecting a suitable method of statistical analysis. Here, the longitudinal study design collected data at five time points, increasing the sample size and power for analysis. By applying MVML modelling, we were able to make full use of the available data, identifying both time-invariant and time-varying factors that influence changes in HrQoL. This demonstrates the applicability of MVML modelling in a hemophilia population and suggests that such methodology may also find application in future studies of longitudinal change in
HrQoL in other chronic/rare disease populations that have long periods of stability with occasional acute exacerbations.

An additional limitation of this study is the measurement of HrQoL at fixed time points, not during an acute event. Despite this, significant changes in HrQoL due to acute events were identified that suggest previous underestimation of the true impact of an acute event on HrQoL. Future studies should attempt to measure HrQoL at or as close as possible to the time of acute events, although this may prove to be a challenge.

Understanding how HrQoL is influenced by both sociodemographic and clinical factors over a prolonged period may help researchers and healthcare providers identify the factors mediating $\mathrm{HrQoL}$ in patients with stable chronic diseases. This would allow providers to tailor patient education and management strategies, allowing for better care management both at the individual patient and disease-population levels.

Acknowledgements: HUGS Va was sponsored by: CSL Behring, Baxter Healthcare Corporation, Bayer Foundation, Novo Nordisk, and Pfizer (formerly Wyeth). Additional financial support was obtained from the Federal Hemophilia Treatment Centers/Region IX, Grifols, Red Chip, and CHOC at Home.

The Hemophilia Utilization Group Study Part Va (HUGS Va): University of Southern California: Michael B. Nichol, PhD (Principal Investigator), Kathleen A. Johnson, PharmD, MPH, PhD (Principal Investigator, deceased), Zheng-Yi Zhou, MS, Mimi Lou, MS, JiatLing Poon, PhD, Xiaoli Niu, Joanne Wu, MS, Michael Goode (Programmer);

Children's Hospital Los Angeles, Hemostasis and Thrombosis Center (CHLA): Cathliyn Buranahirun, PsyD (Site Principal Investigator), Robert Miller (Former Site Principal Investigator), Jennifer Hanley;

Children's Hospital of Orange County, Hemophilia Treatment Center (CHOC): Amit Soni, MD (Site Principal Investigator), Heather Huszti, $\mathrm{PhD}$ (Former Site Principal Investigator), Brandy Fitzhenry, James Fabella;

University of Colorado Denver (UCD): Brenda Riske, MS, MBA, MPA (Site Principal Investigator), Carissa Smith, Cassie Ross, Deirdre Cooper-Blacketer, Julie Smith;

Indiana Hemophilia \& Thrombosis Center, Hemophilia Treatment Center (IHTC): Amy Shapiro, MD (Site Principal Investigator), Natalie Duncan, MPH, Brandy Trawinski, Jayme Harvey, Melissa Meyer;

UMASS Memorial Hospital, New England Hemophilia Center (UMASS): Ann D. Forsberg, MA, MPH (Site Principal Investigator), Patricia Forand, RN.;

University of Texas at Houston, Gulf States Hemophilia and Thrombophilia Center (GSHTC): Megan M. Ullman, MA, MPH (Site Principal Investigator);

HUGS Steering Committee: Randall G. Curtis, MBA, Shelby L. Dietrich, MD, Marion A. Koerper, MD, Brenda Riske, MS, MBA, MPA, Megan M. Ullman, MA, MPH, Heather Huszti, PhD, Judith R. Baker, DPH, MHSA;

Consultants: Jason N. Doctor, PhD, Femida Gwadry-Sridhar, BSPhm, PhD, Denise R. Globe, PhD, Kathy L. Parish, PhD.

Conflict of Interest Disclosure: JL Poon was a graduate fellow with Baxter Biosciences.

JN Doctor has acted as a consultant for Baxter Biosciences.

Corresponding Author: Jiat-Ling Poon, $\mathrm{PhD}$; University of Southern California, School of Pharmacy, 1985 Zonal Ave, PSC 206-BE, Los Angeles, CA 90089, USA (e-mail:jiatlinp@usc.edu). 


\section{REFERENCES}

1. World Federation of Hemophilia. Guidelines for the Management of Hemophilia: World Federation of Hemophilia; 2005.

2. Choiniere M, Melzack R. Acute and chronic pain in hemophilia. Pain. 1987;31(3):317-331.

3. Gringeri A, Mantovani LG, Scalone L, Mannucci PM. Cost of care and quality of life for patients with hemophilia complicated by inhibitors: the COCIS Study Group. Blood. 2003;102(7):2358-2363. doi:10.1182/ blood-2003-03-09412003-03-0941.

4. Lindvall K, Von Mackensen S, Berntorp E. Quality of life in adult patients with haemophilia-a single centre experience from Sweden. Haemophilia. 2012;18(4):527-531. doi:10.1111/j.1365-2516.2012.02765.x.

5. Solovieva S. Clinical severity of disease, functional disability and healthrelated quality of life. Three-year follow-up study of 150 Finnish patients with coagulation disorders. Haemophilia. 2001;7(1):53-63.

6. Zhou ZY, Wu J, Baker J, Curtis R, Forsberg A, Huszti H, et al. Haemophilia utilization group study—Part Va (HUGS Va): design, methods and baseline data. Haemophilia. 2011;17(5):729-736. doi:10.1111/j.1365-2516.2011.02595.x.

7. Poon JL, Zhou ZY, Doctor JN, Wu J, Ullman MM, Ross C, et al. Quality of life in haemophilia A: Hemophilia Utilization Group Study Va (HUGS-Va). Haemophilia. 2012;18(5):699-707. doi:10.1111/j.1365-2516.2012.02791.x.

8. Schick M, Stucki G, Rodriguez M, Meili EO, Huber E, Michel BA, et al. Haemophilic; arthropathy: assessment of quality of life after total knee arthroplasty. Clin Rheumatol. 1999;18(6):468-472.

9. Brown TM, Lee WC, Joshi AV, Pashos CL. Health-related quality of life and productivity impact in haemophilia patients with inhibitors. Haemophilia. 2009;15(4):911-917. doi:10.1111/j.1365-2516.2009.02032.x.

10. Duncan N, Shapiro A, Ye X, Epstein J, Luo MP. Treatment patterns, health-related quality of life and adherence to prophylaxis among haemophilia A patients in the United States. Haemophilia. 2012;18(5):760765. doi:10.1111/j.1365-2516.2012.02813.x.

11. Ware J Jr, Kosinski M, Keller SD. A 12-Item Short-Form Health Survey: construction of scales and preliminary tests of reliability and validity. Med Care. 1996;34(3):220-233.

12. Varni JW, Seid M, Kurtin PS. PedsQL 4.0: reliability and validity of the Pediatric Quality of Life Inventory version 4.0 generic core scales in healthy and patient populations. Med Care. 2001;39(8):800-812.

13. Varni JW, Limbers CA, Burwinkle TM. Impaired health-related quality of life in children and adolescents with chronic conditions: a comparative analysis of 10 disease clusters and 33 disease categories/severities utilizing the PedsQL 4.0 Generic Core Scales. Health Qual Life Outcomes. 2007:5:43.

14. Varni JW, Limbers CA, Burwinkle TM. Parent proxy-report of their children's health-related quality of life: an analysis of 13,878 parents' reliability and validity across age subgroups using the PedsQL 4.0 Generic Core Scales. Health Qual Life Outcomes. 2007;5:2.

15. Bryk AS, Raudenbush Sw. Application of Hierarchical Linear-Models to Assessing Change. Psychol Bull. 1987;101(1):147-158. doi:10.1037/ 0033-2909.101.1.147.

16. MacCallum RC, Kim C, Malarkey WB, KiecoltGlaser JK. Studying multivariate change using multilevel models and latent curve models. Multivar Behav Res. 1997;32(3):215-253. doi:10.1207/ s15327906mbr3203_1.

17. Curran PJ. Have multilevel models been structural equation models all along? Multivar Behav Res. 2003;38(4):529-568. doi:10.1207/ s15327906mbr3804_5.

18. Raghunathan TE, Lepkowski JM, Van Hoewyk J, Solenberger P. A Multivariate Technique for Multiply Imputing Missing Values Using a Sequence of Regression Models. Surv Methodol. 2001;27(1):85-95. 